\title{
Perfect Reconciliation in Quantum Key Distribution with Order-Two Frames
}

\author{
Luis A. Lizama-Perez ${ }^{10000-0001-5109-2927]}$ and José Mauricio López Romero² \\ Sección de Posgrado de la Universidad Politécnica de Pachuca, \\ Ex-Hacienda de Santa Bárbara, 43830, México \\ luislizama@upp.edu.mx \\ Cinvestav Querétaro, Libramiento Norponiente 2000, \\ Real de Juriquilla, 76230, Santiago de Querétaro, Querétaro, México \\ jm.lopez@cinvestav.mx
}

\begin{abstract}
We present an error reconciliation method for Quantum Key Distribution (QKD) that corrects $100 \%$ of errors generated in regular binary frames transmitted over a noisy quantum channel regardless of the quantum channel error rate. In a previous investigation, we introduced a novel distillation QKD algorithm whose secret key rate descends linearly with respect to the channel error rate. Now, as the main achievement of this work, we demonstrate an improved algorithm capable of retaining almost all the secret information enclosed in the regular binary frames. Remarkably, this technique increases quadratically the secret key rate as a function of the double matching detection events and doubly quadratically in the number of the quantum pulses. Furthermore, this reconciliation method opens up the opportunity to use less attenuated quantum pulses, would allow greater QKD distances at drastically increased secret key rate. Since our method can be implemented as a software update, we hope that quantum key distribution technology would be fast deployed over global data networks in the quantum era.
\end{abstract}

Keywords: QKD · distillation · reconciliation

\section{Introduction}

The arrival of the quantum era and quantum computers in the short term is imminent. One of the most profound consequences of the quantum era is that the security of digital data as we know it today must be radically changed due to the power of computers to break the security of asymmetric key cryptographic methods and at the same time must increase the sizes of the symmetrical keys.

Countermeasures to address the threat of quantum computers have been led by NIST, which launched a selection process for new cryptographic algorithms for the quantum era in 2017 [1]. However, it is to be expected that it will take years to implement and technologically adapt the new methods to be used in global data networks [2].

Fortunately, the quantum cryptographic key distribution (QKD) is a post-quantum scheme that appeared almost four decades ago that has been widely evaluated and discussed by the scientific community. In addition, QKD can be implemented through satellite links or already installed fiber optic networks. Particularly, we have introduced new QKD protocols to overcome quantum hacking attacks $[3,4]$.

However, the QKD suffers from a serious challenge: the noise of the quantum channel and the errors in the transmitted information that it produces, imposes a severe limitation in terms of the length of the link and the speed of the secret bits that can be derived [5]. 
Reconciliation methods developed natively for QKD are BBBSS [6] based on binary search, Cascade $[7,8]$ that uses binary search and backtracking, but they are based on the parity computation of the received information blocks and do not take advantage of the properties of communication with quantum states; instead, it is highly interactive, requiring multiple rounds of correction of bits.

In view of the above, it has been necessary to resort to other reconciliation techniques developed in the field of data communications. Reconciliation methods based on error correcting codes are Winnow $[9,10,11]$ which uses parity check and Hamming error correction code. It corrects one error per block, so the choice of block length is very sensitive because additional errors may be introduced if a block contains two or more errors [12]. Also, Forward Error Correction (FEC) is used to achieve reconciliation as the discrete number of Low-Density Parity-Check (LDPC) codes. However, LDPC has the disadvantage that requires redundant information that must be transmitted along the information data $[13,14,15,16,17]$. Recently, polar encoding has emerged as an encoding method in finding error correction codes that are close to the Shannon limit $[18,19,20]$. Beyond the mentioned drawbacks, none of these schemes is capable of handling a quantum channel error rate beyond $25 \%$ [21].

We published in $[22,23]$ a new reconciliation algorithm that takes advantage of the characteristics of quantum communication, which, simply put, is equivalent to having two classical communication channels, one in the quantum $\mathbf{X}$ basis and the other in the $\mathbf{Z}$ basis. By means of a reverse reconciliation process, Bob sends parity information from these two channels so that Alice is able to recognize Bob's chosen bases on which the secret information is encoded as depicted in Figure 1.

\section{Alice's frame}

$$
\left(\begin{array}{cc}
0_{\mathrm{x}} & 1_{\mathrm{z}} \\
1_{\mathrm{x}} & 1_{\mathrm{z}}
\end{array}\right) \longrightarrow\left(\begin{array}{c}
0_{\mathrm{x}}- \\
-1_{\mathrm{z}}
\end{array}\right)
$$

Fig. 1: General scheme of frame-based QKD approach. Each row of the frame is a pair of non-orthogonal states: each position inside the frame encodes a quantum bit, so in the first position is $\left|i_{X}\right\rangle$ or $\left|(1-i)_{X}\right\rangle$ while in the second $\left|i_{Z}\right\rangle$ or $\left|(1-i)_{Z}\right\rangle$ where $i=0,1$.

A two order frame, as it can be seen in Figure 1, is a $2 \times 2$ matrix, structured by two rows and two columns. One row represents a pair of non-orthogonal states where the first column of the frame contains the base $\mathbf{X}$ encoded quantum bit $\left|i_{X}\right\rangle$ or $\left|(1-i)_{X}\right\rangle$ and the second column the base $\mathbf{Z}$ quantum bit, that is $\left|i_{Z}\right\rangle$ or $\left|(1-i)_{Z}\right\rangle$ where $i=0,1$. Once Bob measures a pair of non-orthogonal states and provided he gets a DMDE (Double Matching Detection Event), he obtains the encoded bit in the first or second column of the first row of the frame, as shown in Figures 1 and 2. Bob's frame is complete once he gets the second DMDE. 


\section{Alice's two pairs of non-orthogonal states}

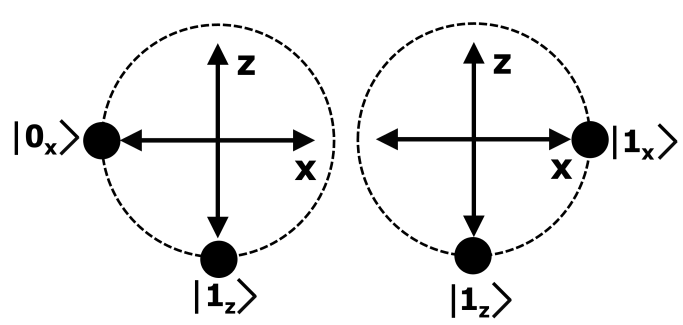

Bob's double matching detection

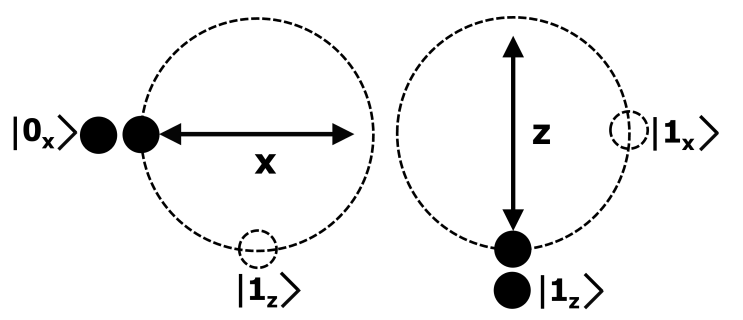

Fig. 2: Alice sends two pairs of non-orthogonal states to Bob over the quantum channel: $\left(\left|0_{X}\right\rangle,\left|1_{Z}\right\rangle\right)$ and $\left(\left|1_{X}\right\rangle,\left|1_{Z}\right\rangle\right)$. Bob's gets two events of double matching detection events: $\left(\left|0_{X}\right\rangle,+\right.$ and $\left(+,\left|1_{Z}\right\rangle\right)$

In fact, the two rows of a frame are not received sequentially, instead Bob must inform Alice about the DMDE that he obtained, then Alice responds to Bob how to arrange the rows to construct the frames as illustrated in the message exchange in Figure 3. For this reason, each DMDE is labelled by an index that has the form (CSS, $i_{1}, i_{2}$ ) where $i_{1}$ is the number of the first NO-QP and $i_{2}$ is the number of the second NO-QP. The index is assigned during transmission, so is known by Alice and Bob, CSS will be explained shortly.

This article is focused on the discussion and explanation of the reconciliation method, rather than on a detailed discussion of the attacks over the system. So, Section 2 contains a detailed discussion of the reconciliation method. In Section 3 we derive the relation for the secret throughput of the frame-based method. Finally, section 4 contains a brief discussion about the main quantum attacks. But before going to the details of the reconciliation method, let us succinctly state the research problem and the general idea of the new method.

Research problem statement. The reconciliation method must be able to detect the errors produced into the pairs of non-orthogonal quantum states $\left(\overrightarrow{\mathbf{0}_{\mathbf{X}}}, \mathbf{0}_{\mathbf{z}}\right),\left(\mathbf{0}_{\mathbf{X}}, \mathfrak{\mathbf { 0 }}_{\mathbf{z}}\right),\left(\overrightarrow{\mathbf{0}_{\mathbf{X}}}, \mathbf{1}_{\mathbf{z}}\right),\left(\mathbf{0}_{\mathbf{X}}, \mathbf{1}_{\mathbf{z}}\right)$, $\left(\mathfrak{1}_{\mathbf{X}}, \mathbf{0}_{\mathbf{Z}}\right),\left(\mathbf{1}_{\mathbf{X}}, \mathfrak{0}_{\mathbf{Z}}\right),\left(\mathfrak{1}_{\mathbf{X}}, \mathbf{1}_{\mathbf{Z}}\right)$ and $\left(\mathbf{1}_{\mathbf{X}}, \mathfrak{1}_{\mathbf{Z}}\right)$ where the overbracket symbol $\boldsymbol{n}$ represents the error that is produced in a transmitted NO-QP. We argue that if we can detect all these types of errors, we will achieve error correction that is invariant with respect to the error rate of the quantum channel. The following types of frames (which have been enumerated according to [22]) will be used:

1. Auxiliary frames, of two types, null and unitary frames as well as their conjugate denoted with a math apostrophe. We call $f_{7}$ the null frame while $f_{11}$ is the unitary frame:

$$
f_{7}=\left(\begin{array}{ll}
\mathbf{0}_{\mathbf{X}} & \mathbf{0}_{\mathbf{Z}} \\
\mathbf{0}_{\mathbf{X}} & \mathbf{0}_{\mathbf{Z}}
\end{array}\right) f_{7}{ }^{\prime}=\left(\begin{array}{ll}
\mathbf{1}_{\mathbf{X}} & \mathbf{1}_{\mathbf{Z}} \\
\mathbf{1}_{\mathbf{X}} & \mathbf{1}_{\mathbf{Z}}
\end{array}\right) f_{11}=\left(\begin{array}{ll}
\mathbf{1}_{\mathbf{X}} & \mathbf{1}_{\mathbf{Z}} \\
\mathbf{1}_{\mathbf{X}} & \mathbf{1}_{\mathbf{Z}}
\end{array}\right) f_{11}{ }^{\prime}=\left(\begin{array}{ll}
\mathbf{0}_{\mathbf{X}} & \mathbf{0}_{\mathbf{Z}} \\
\mathbf{0}_{\mathbf{X}} & \mathbf{0}_{\mathbf{Z}}
\end{array}\right)
$$


2. Regular-frames (twelve types) and their conjugate:

$$
\begin{aligned}
& f_{1}=\left(\begin{array}{ll}
\mathbf{0}_{\mathbf{X}} & \mathbf{1}_{\mathbf{Z}} \\
\mathbf{1}_{\mathbf{X}} & \mathbf{0}_{\mathbf{Z}}
\end{array}\right) \quad f_{1}{ }^{\prime}=\left(\begin{array}{ll}
\mathbf{1}_{\mathbf{X}} & \mathbf{0}_{\mathbf{Z}} \\
\mathbf{0}_{\mathbf{X}} & \mathbf{1}_{\mathbf{Z}}
\end{array}\right) \quad f_{5}=\left(\begin{array}{ll}
\mathbf{1}_{\mathbf{X}} & \mathbf{0}_{\mathbf{Z}} \\
\mathbf{0}_{\mathbf{X}} & \mathbf{1}_{\mathbf{Z}}
\end{array}\right) \quad f_{5}{ }^{\prime}=\left(\begin{array}{ll}
\mathbf{0}_{\mathbf{X}} & \mathbf{1}_{\mathbf{Z}} \\
\mathbf{1}_{\mathbf{X}} & \mathbf{0}_{\mathbf{Z}}
\end{array}\right) \\
& f_{2}=\left(\begin{array}{ll}
\mathbf{1}_{\mathbf{X}} & \mathbf{0}_{\mathbf{Z}} \\
\mathbf{1}_{\mathbf{X}} & \mathbf{1}_{\mathbf{Z}}
\end{array}\right) \quad f_{2}{ }^{\prime}=\left(\begin{array}{ll}
\mathbf{0}_{\mathbf{X}} & \mathbf{1}_{\mathbf{Z}} \\
\mathbf{0}_{\mathbf{X}} & \mathbf{0}_{\mathbf{Z}}
\end{array}\right) \quad f_{6}=\left(\begin{array}{ll}
\mathbf{1}_{\mathbf{X}} & \mathbf{1}_{\mathbf{Z}} \\
\mathbf{1}_{\mathbf{X}} & \mathbf{0}_{\mathbf{Z}}
\end{array}\right) \quad f_{6}{ }^{\prime}=\left(\begin{array}{ll}
\mathbf{0}_{\mathbf{X}} & \mathbf{0}_{\mathbf{Z}} \\
\mathbf{0}_{\mathbf{X}} & \mathbf{1}_{\mathbf{Z}}
\end{array}\right) \\
& f_{3}=\left(\begin{array}{ll}
\mathbf{0}_{\mathbf{X}} & \mathbf{1}_{\mathbf{Z}} \\
\mathbf{1}_{\mathbf{X}} & \mathbf{1}_{\mathbf{Z}}
\end{array}\right) \quad f_{3}{ }^{\prime}=\left(\begin{array}{ll}
\mathbf{1}_{\mathbf{X}} & \mathbf{0}_{\mathbf{Z}} \\
\mathbf{0}_{\mathbf{X}} & \mathbf{0}_{\mathbf{Z}}
\end{array}\right) \quad f_{4}=\left(\begin{array}{ll}
\mathbf{1}_{\mathbf{X}} & \mathbf{1}_{\mathbf{Z}} \\
\mathbf{0}_{\mathbf{X}} & \mathbf{1}_{\mathbf{Z}}
\end{array}\right) \quad f_{4}{ }^{\prime}=\left(\begin{array}{ll}
\mathbf{0}_{\mathbf{X}} & \mathbf{0}_{\mathbf{Z}} \\
\mathbf{1}_{\mathbf{X}} & \mathbf{0}_{\mathbf{Z}}
\end{array}\right) \\
& f_{9}=\left(\begin{array}{ll}
\mathbf{0}_{\mathbf{X}} & \mathbf{1}_{\mathbf{Z}} \\
\mathbf{0}_{\mathbf{X}} & \mathbf{0}_{\mathbf{Z}}
\end{array}\right) \quad f_{9}{ }^{\prime}=\left(\begin{array}{ll}
\mathbf{1}_{\mathbf{X}} & \mathbf{0}_{\mathbf{Z}} \\
\mathbf{1}_{\mathbf{X}} & \mathbf{1}_{\mathbf{Z}}
\end{array}\right) \quad f_{10}=\left(\begin{array}{ll}
\mathbf{1}_{\mathbf{X}} & \mathbf{0}_{\mathbf{Z}} \\
\mathbf{0}_{\mathbf{X}} & \mathbf{0}_{\mathbf{Z}}
\end{array}\right) \quad f_{10}{ }^{\prime}=\left(\begin{array}{ll}
\mathbf{0}_{\mathbf{X}} & \mathbf{1}_{\mathbf{Z}} \\
\mathbf{1}_{\mathbf{X}} & \mathbf{1}_{\mathbf{Z}}
\end{array}\right) \\
& f_{8}=\left(\begin{array}{ll}
\mathbf{0}_{\mathbf{X}} & \mathbf{0}_{\mathbf{Z}} \\
\mathbf{1}_{\mathbf{X}} & \mathbf{1}_{\mathbf{Z}}
\end{array}\right) \quad f_{8}=\left(\begin{array}{ll}
\mathbf{1}_{\mathbf{X}} & \mathbf{1}_{\mathbf{Z}} \\
\mathbf{0}_{\mathbf{X}} & \mathbf{0}_{\mathbf{Z}}
\end{array}\right) \quad f_{12}=\left(\begin{array}{ll}
\mathbf{1}_{\mathbf{X}} & \mathbf{1}_{\mathbf{Z}} \\
\mathbf{0}_{\mathbf{X}} & \mathbf{0}_{\mathbf{Z}}
\end{array}\right) \quad f_{12}{ }^{\prime}=\left(\begin{array}{ll}
\mathbf{0}_{\mathbf{X}} & \mathbf{0}_{\mathbf{Z}} \\
\mathbf{1}_{\mathbf{X}} & \mathbf{1}_{\mathbf{Z}}
\end{array}\right) \\
& f_{13}=\left(\begin{array}{ll}
\mathbf{0}_{\mathbf{X}} & \mathbf{0}_{\mathbf{Z}} \\
\mathbf{0}_{\mathbf{X}} & \mathbf{1}_{\mathbf{Z}}
\end{array}\right) f_{13}=\left(\begin{array}{ll}
\mathbf{1}_{\mathbf{X}} & \mathbf{1}_{\mathbf{Z}} \\
\mathbf{1}_{\mathbf{X}} & \mathbf{0}_{\mathbf{Z}}
\end{array}\right) \quad f_{14}=\left(\begin{array}{ll}
\mathbf{0}_{\mathbf{X}} & \mathbf{0}_{\mathbf{Z}} \\
\mathbf{1}_{\mathbf{X}} & \mathbf{0}_{\mathbf{Z}}
\end{array}\right) \quad f_{14}{ }^{\prime}=\left(\begin{array}{ll}
\mathbf{1}_{\mathbf{X}} & \mathbf{1}_{\mathbf{Z}} \\
\mathbf{0}_{\mathbf{X}} & \mathbf{1}_{\mathbf{Z}}
\end{array}\right)
\end{aligned}
$$

The frame $f_{15}=\left(\begin{array}{ll}\mathbf{0}_{\mathbf{X}} & \mathbf{1}_{\mathbf{Z}} \\ \mathbf{0}_{\mathbf{X}} & \mathbf{1}_{\mathbf{Z}}\end{array}\right)$ and the frame $f_{16}=\left(\begin{array}{ll}\mathbf{1}_{\mathbf{X}} & \mathbf{0}_{\mathbf{Z}} \\ \mathbf{1}_{\mathbf{X}} & \mathbf{0}_{\mathbf{Z}}\end{array}\right)$ are not used in this context. Bob obtains the conjugate frames by inverting the measured bits so they are not obtained from the channel measurements.

General idea of the method. It is our goal to demonstrate that using the Composed Sifting String (CSS) is possible that Alice reconciliate 100\% of the errors produced in received Bob's DMDE.

- Just to bring it in context, the Sifting String (SS) as stated in [22] is constructed using the sifting bits and the measured bits into Bob's frames. The sifting bits are obtained applying the XOR function to the bits within the columns (from the left to the right column) of Bob's frames, where a vacuum bit is taken as a zero bit. The measured bits are taken directly from the bits inside Bob's frame. This is so because the secret bit is derived from the final configuration of Bob's frames, that we call Measurement Results (MR) as represented in Table 1. The sifting bits are written first into SS while the measured bits are placed next:

$$
\mathrm{SS}=1^{\text {st }} \text { sifting bit } \| 2^{\text {nd }} \text { sifting bit, } 1^{\text {st }} \text { measured bit } \| 2^{\text {nd }} \text { measured bit }
$$

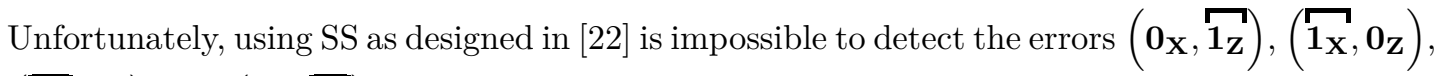
$\left(\mathbf{1}_{\mathbf{X}}, \mathbf{1}_{\mathbf{z}}\right)$ and $\left(\mathbf{1}_{\mathbf{X}}, \mathfrak{1}_{\mathbf{z}}\right)$.

- Now, in this new reconciliation method we introduce the Composed Sifting String (CSS) which is constructed taken the sifting bits of Bob's frame but also the sifting bits of Bob's conjugate frame, that is:

$$
\begin{aligned}
\mathrm{CSS}= & 1^{\text {st }} \text { sifting bit } \| 2^{\text {nd }} \text { sifting bit } \| \\
& 1^{\text {st }} \text { sifting bit of conjugate frame } \| 2^{\text {nd }} \text { sifting bit of conjugate frame }
\end{aligned}
$$


We will demonstrate that using CSS and without compromising the security of the scheme, is

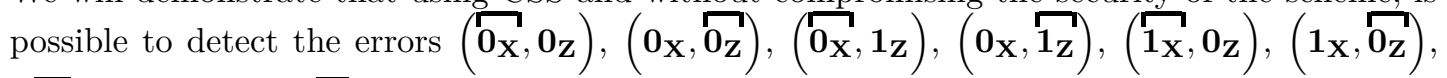
$\left(\overparen{\mathbf{1}}_{\mathbf{X}}, \mathbf{1}_{\mathbf{z}}\right)$ and $\left(\mathbf{1}_{\mathbf{X}}, \overparen{1}_{\mathbf{z}}\right)$.

\section{Perfect Reconciliation Using Order-Two Binary Frames}

We begin this section by establishing the general steps of the reconciliation method which we will justify throughout the section. To simplify notation, throughout this document we will represent a quantum state using a bold letter instead of the usual ket notation, so we denote $\left|i_{X}\right\rangle$ as $\mathbf{i}_{\mathbf{X}}$.

1. Alice creates NO-QPL (the Non-Orthogonal Quantum Pair List) and sends, one by one, each NO-QP (the Non-Orthogonal Quantum Pair) across QC (the Quantum Channel). NO-QP can be $\left(\mathbf{i}_{\mathbf{X}}, \mathbf{i}_{\mathbf{Z}}\right)$ or $\left(\mathbf{i}_{\mathbf{X}},(\mathbf{1}-\mathbf{i}) \mathbf{Z}\right)$ where $i=0,1$.

2. Bob chooses randomly the measurement basis: $\mathbf{X}$ or $\mathbf{Z}$, that he will use to measure both states inside NO-QP. After Bob registers DDE (the Double Detection Events) he sends DDEL (the Double Detection Event List) to Alice.

3. Alice receive DDEL from QC, she creates FAIL (the Frame Arrangement Information List) and sends it to Bob.

4. Bob receives FAIL and he computes CSSL (the Composed Sifting String List). Then he returns CSSL to Alice.

5. Alice detect errors and identifies MR in regular frames. Alice sends FDL (the Frame to Delete List) to Bob.

6. Bob eliminates the frames indicated in FDL, then he creates SeS using MRT as written in Table 1.

We call step 3 of the protocol privacy pre-amplification, in this step Alice performs all the combinations of the DMDE to form the frames that she is going to use in order to successfully carry out the error correction process. Then, the number of possible frames is given by the combination formula $\left(\begin{array}{l}n \\ 2\end{array}\right)=\frac{n !}{2 !(n-2) !}$ where $n$ is the number of DMDE. The general diagram showing the protocol message exchange is shown in Figure 3.

Table 1: Matching Results Table (MRT) for $2 \times 2$ frames.

$$
\begin{aligned}
& \operatorname{MR}=00\left(\begin{array}{cc}
\left|\bullet_{X}\right\rangle & - \\
\left|\bullet_{X}\right\rangle & -
\end{array}\right) \quad \operatorname{MR}=10\left(\begin{array}{cc}
\left|\bullet_{X}\right\rangle & - \\
- & \left|\bullet_{z}\right\rangle
\end{array}\right) \\
& \mathrm{MR}=01\left(\begin{array}{cc}
- & \left|\bullet_{z}\right\rangle \\
- & \left|\bullet_{z}\right\rangle
\end{array}\right) \quad \operatorname{MR}=11\left(\begin{array}{c}
-\left|\bullet_{z}\right\rangle \\
\left|\bullet_{X}\right\rangle
\end{array}\right)
\end{aligned}
$$




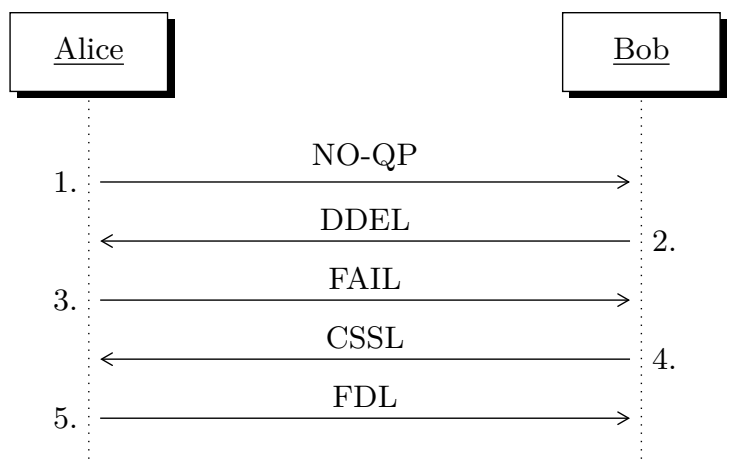

Fig. 3: The reconciliation message exchange: NO-QP represents the quantum pulses over the quantum channel. Remaining steps take place over the classical channel.

\subsection{Regular and Conjugate $2 \times 2$ Frames}

Conjugate frames are derived from regular frames thus they are not obtained from the physical quantum channel. They are used just to derive a useful complementary set of sifting bits. Below we will show each one of the regular frames, each one with its respective conjugated frame and we will add its corresponding CSS each MR.

$$
\begin{aligned}
& \left(\begin{array}{cc}
\mathbf{0}_{\mathbf{X}} & \mathbf{1}_{\mathbf{Z}} \\
\mathbf{1}_{\mathbf{X}} & \mathbf{0}_{\mathbf{Z}}
\end{array}\right): \underset{01}{\left(\begin{array}{c}
+\mathbf{1}_{\mathbf{Z}} \\
+\mathbf{0}_{\mathbf{Z}}
\end{array}\right)} \underset{0}{\left(\begin{array}{c}
\mathbf{0}_{\mathbf{X}}+ \\
\mathbf{1}_{\mathbf{X}}+
\end{array}\right)} \underset{00}{\left(\begin{array}{c}
\mathbf{0}_{\mathbf{X}}+ \\
+\mathbf{0}_{\mathbf{Z}}
\end{array}\right)} \underset{10}{\left(\begin{array}{c}
+\mathbf{1}_{\mathbf{Z}} \\
\mathbf{1}_{\mathbf{X}}+
\end{array}\right)} \\
& \left(\begin{array}{cc}
\mathbf{1}_{\mathbf{X}} & \mathbf{0}_{\mathbf{Z}} \\
\mathbf{0}_{\mathbf{X}} & \mathbf{1}_{\mathbf{Z}}
\end{array}\right): \underset{01}{\left(\begin{array}{c}
+\mathbf{0}_{\mathbf{Z}} \\
+\mathbf{1}_{\mathbf{Z}}
\end{array}\right)} \underset{10}{\left(\begin{array}{c}
\mathbf{1}_{\mathbf{X}}+ \\
\mathbf{0}_{\mathbf{X}}+
\end{array}\right)} \underset{11}{\left(\begin{array}{c}
\mathbf{1}_{\mathbf{X}}+ \\
+\mathbf{1}_{\mathbf{Z}}
\end{array}\right)} \underset{00}{\left(\begin{array}{c}
+\mathbf{0}_{\mathbf{Z}} \\
\mathbf{0}_{\mathbf{X}}+
\end{array}\right)} \\
& \begin{array}{lllll}
\text { CSS : } & 0101 & 1010 & 0011 & 1100
\end{array}
\end{aligned}
$$

$$
\begin{aligned}
& \left(\begin{array}{cc}
\mathbf{1}_{\mathbf{X}} \mathbf{0}_{\mathbf{Z}} \\
\mathbf{1}_{\mathbf{X}} \mathbf{1}_{\mathbf{Z}}
\end{array}\right): \underset{01}{\left(\begin{array}{c}
+\mathbf{0}_{\mathbf{Z}} \\
+\mathbf{1}_{\mathbf{Z}}
\end{array}\right)} \underset{00}{\left(\begin{array}{c}
\mathbf{1}_{\mathbf{X}}+ \\
\mathbf{1}_{\mathbf{X}}+
\end{array}\right)} \underset{11}{\left(\begin{array}{c}
\mathbf{1}_{\mathbf{X}}+ \\
+\mathbf{1}_{\mathbf{Z}}
\end{array}\right)} \underset{10}{\left(\begin{array}{c}
+\mathbf{0}_{\mathbf{Z}} \\
\mathbf{1}_{\mathbf{X}}+
\end{array}\right)}
\end{aligned}
$$

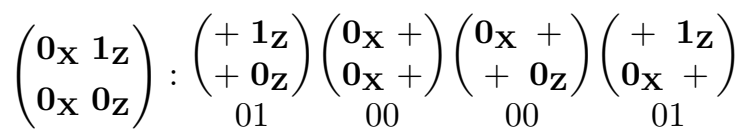

$$
\begin{aligned}
& \begin{array}{lllll}
\text { CSS : } & 0101 & 0000 & 1100 & 1001
\end{array}
\end{aligned}
$$




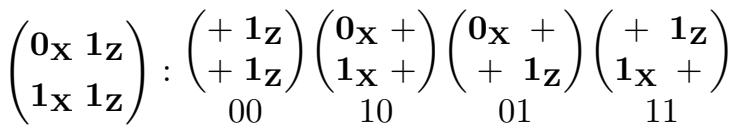

$$
\begin{aligned}
& \left(\begin{array}{ll}
\mathbf{1}_{\mathbf{X}} & \mathbf{0}_{\mathbf{Z}} \\
\mathbf{0}_{\mathbf{X}} & \mathbf{0}_{\mathbf{Z}}
\end{array}\right):\left(\begin{array}{c}
+\mathbf{0}_{\mathbf{Z}} \\
+\mathbf{0}_{\mathbf{Z}}
\end{array}\right)\left(\begin{array}{c}
\mathbf{1}_{\mathbf{X}}+ \\
\mathbf{0}_{\mathbf{X}}+
\end{array}\right)\left(\begin{array}{c}
\mathbf{1}_{\mathbf{X}}+ \\
+\mathbf{0}_{\mathbf{Z}}
\end{array}\right) \underset{10}{\left(\begin{array}{c}
+ \\
\mathbf{0}_{\mathbf{X}}+
\end{array}\right)} \\
& \begin{array}{lllll}
\text { CSS : } & 0000 & 1010 & 0110 & 1100
\end{array}
\end{aligned}
$$

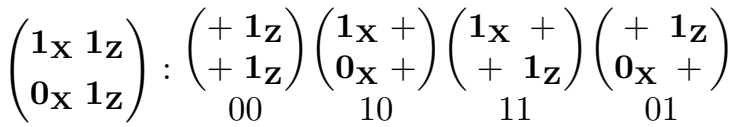

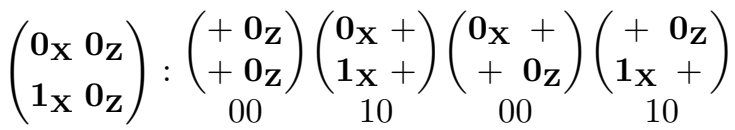

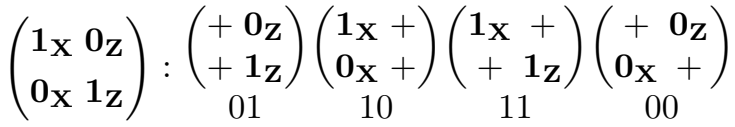

$$
\begin{aligned}
& \left(\begin{array}{ll}
\mathbf{0}_{\mathbf{X}} & \mathbf{1}_{\mathbf{Z}} \\
\mathbf{1}_{\mathbf{X}} & \mathbf{0}_{\mathbf{Z}}
\end{array}\right):\left(\begin{array}{c}
+\mathbf{1}_{\mathbf{Z}} \\
+\mathbf{0}_{\mathbf{Z}}
\end{array}\right)\left(\begin{array}{c}
\mathbf{0}_{\mathbf{X}}+ \\
\mathbf{1}_{\mathbf{X}}+
\end{array}\right)\left(\begin{array}{c}
\mathbf{0}_{\mathbf{X}}+ \\
+ \\
+\mathbf{0}_{\mathbf{Z}}
\end{array}\right)\left(\begin{array}{c}
+\mathbf{1}_{\mathbf{Z}} \\
\mathbf{1}_{\mathbf{X}}+
\end{array}\right) \\
& \text { CSS : } \quad 0101 \quad 1010 \quad 1100 \quad 0011
\end{aligned}
$$

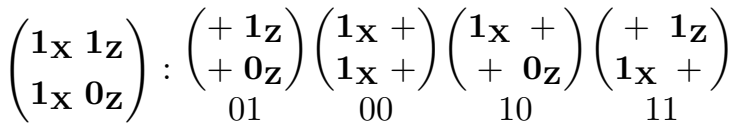

$$
\begin{aligned}
& \left(\begin{array}{cc}
\mathbf{0}_{\mathbf{X}} & \mathbf{0}_{\mathbf{Z}} \\
\mathbf{0}_{\mathbf{X}} & \mathbf{1}_{\mathbf{Z}}
\end{array}\right): \underset{01}{\left(\begin{array}{c}
+\mathbf{0}_{\mathbf{Z}} \\
+\mathbf{1}_{\mathbf{Z}}
\end{array}\right)} \underset{00}{\left(\begin{array}{c}
\mathbf{0}_{\mathbf{X}}+ \\
\mathbf{0}_{\mathbf{X}}+
\end{array}\right)} \underset{01}{\left(\begin{array}{c}
\mathbf{0}_{\mathbf{X}}+ \\
+ \\
\mathbf{1}_{\mathbf{Z}}
\end{array}\right)} \underset{00}{\left(\begin{array}{c}
+\mathbf{0}_{\mathbf{Z}} \\
\mathbf{0}_{\mathbf{X}}+
\end{array}\right)} \\
& \text { CSS : } \quad 0101 \quad 0000 \quad 1001 \quad 1100
\end{aligned}
$$

The sifting algorithm can also be applied to the remaining regular frames $f_{8}, f_{12}, f_{9}, f_{10}, f_{14}$, $f_{14}$ which we do not show here to facilitate the exposition of the method. The security property or frame-based model states that each CSS must map to at least two MR because the secret bit is derived from MR. The results are presented in Table 2. As can be seen there, the cases CSS 1010 and 0101 should be removed because they map a single MR: 00 and 01, respectively. 
Table 2: Each Composed Sifting String (CSS) must be correlated at least to two Matching Results (MR).

The symbol sb denotes the secret bit.

\begin{tabular}{ccccc} 
CSS & frame & MR & sb & action \\
\hline \multirow{2}{*}{0110} & $f_{3}, f_{8}, f_{13}$ & 10 & 0 & distill \\
& $f_{4}, f_{12}, f_{9}$ & 11 & 1 & \\
\hline \multirow{2}{*}{1001} & $f_{2}, f_{8}, f_{14}$ & 11 & 0 & \multirow{2}{*}{ distill } \\
& $f_{6}, f_{12}, f_{10}$ & 10 & 1 & \\
\hline \multirow{2}{*}{0011} & $f_{1}, f_{9}, f_{14}$ & 10 & 0 & distill \\
& $f_{5}, f_{10}, f_{13}$ & 11 & 1 & \\
\hline \multirow{2}{*}{0000} & $f_{2}, f_{6}, f_{9}, f_{13}$ & 00 & 0 & \multirow{2}{*}{ distill } \\
& $f_{3}, f_{4}, f_{10}, f_{14}$ & 01 & 1 & \\
\hline \multirow{2}{*}{1100} & $f_{1}, f_{3}, f_{6}$ & 11 & 0 & \multirow{2}{*}{ distill } \\
& $f_{2}, f_{4}, f_{5}$ & 10 & 1 & \\
\multirow{2}{*}{1010} & $f_{1}, f_{3}, f_{4}, f_{5}$ & 00 & - & remove \\
& $f_{8}, f_{12}, f_{10}, f_{14}$ & 00 & & \\
\hline \multirow{2}{*}{0101} & $f_{1}, f_{2}, f_{5}, f_{6}$ & \multirow{2}{*}{01} & - & remove \\
& $f_{8}, f_{12}, f_{9}, f_{13}$ & & & \\
\hline & & & &
\end{tabular}

Now, we proceed to demonstrate which errors can be detected inside a frame. Due to their structure, is convenient to see the frames grouped as: $\left\{f_{1}, f_{5}\right\},\left\{f_{3}, f_{4}\right\},\left\{f_{2}, f_{6}\right\},\left\{f_{9}, f_{10}\right\},\left\{f_{8}\right.$, $\left.f_{12}\right\}$. In the following equations, the top line contains the frame under MR while the second line shows the conjugated frame. The bottom line hold the computed CSS in each case. The error detection illustrated depends on an error-free NO-QP, so we will solve this point as the error correction pre-processing.

1. The error $\left(\mathbf{1}_{\mathbf{X}}, \mathbf{0}_{\mathbf{Z}}\right)$ is detected with an error-free NO-QP $\left(\mathbf{1}_{\mathbf{X}}, \mathbf{1}_{\mathbf{Z}}\right)$, as the second row in $f_{2}$ because the CSS that is produced by the error is none of the possible error-free CSS.

$$
\begin{aligned}
& \left(\begin{array}{cc}
\mathbf{1}_{\mathbf{X}} \mathbf{0}_{\mathbf{Z}} \\
\mathbf{1}_{\mathbf{X}} \mathbf{1}_{\mathbf{Z}}
\end{array}\right): \underset{01}{\left(\begin{array}{c}
+\mathbf{0}_{\mathbf{Z}} \\
+\mathbf{1}_{\mathbf{Z}}
\end{array}\right)} \underset{00}{\left(\begin{array}{c}
\mathbf{1}_{\mathbf{X}}+ \\
\mathbf{1}_{\mathbf{X}}+
\end{array}\right)} \underset{11}{\left(\begin{array}{c}
\mathbf{1}_{\mathbf{X}}+ \\
+\mathbf{1}_{\mathbf{Z}}
\end{array}\right)} \underset{10}{\left(\begin{array}{c}
+\mathbf{0}_{\mathbf{Z}} \\
\mathbf{1}_{\mathbf{X}}+
\end{array}\right)} \\
& \underset{01}{\left(\begin{array}{cc}
\mathbf{0}_{\mathbf{X}} & \mathbf{1}_{\mathbf{Z}} \\
\mathbf{0}_{\mathbf{X}} & \mathbf{0}_{\mathbf{Z}}
\end{array}\right)} \underset{00}{\left(\begin{array}{c}
+\mathbf{1}_{\mathbf{Z}} \\
+\mathbf{0}_{\mathbf{Z}}
\end{array}\right)} \underset{00}{\left(\begin{array}{c}
\mathbf{0}_{\mathbf{X}}+ \\
\mathbf{0}_{\mathbf{X}}+
\end{array}\right)} \underset{01}{\left(\begin{array}{c}
\mathbf{0}_{\mathbf{X}}+ \\
+\mathbf{0}_{\mathbf{Z}}
\end{array}\right)} \underset{0}{\left(\begin{array}{c}
+\mathbf{1}_{\mathbf{Z}} \\
\mathbf{0}_{\mathbf{X}}+
\end{array}\right)} \\
& \text { CSS : } \quad 0101 \quad 0000 \quad 1100 \quad 1001
\end{aligned}
$$

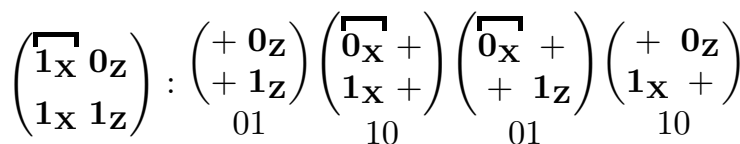

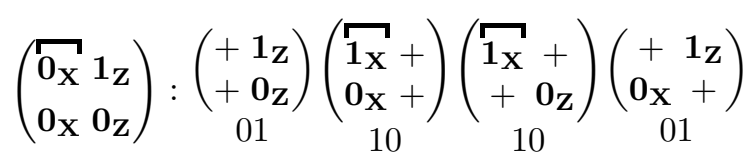

$$
\begin{aligned}
& \text { CSS : } 0101 \quad 1010 \quad 0110 \quad 1001
\end{aligned}
$$


2. The error $\left(\mathbf{1}_{\mathbf{X}}, \mathbf{0}_{\mathbf{Z}}\right)$ is detected with an error-free NO-QP $\left(\mathbf{1}_{\mathbf{X}}, \mathbf{1}_{\mathbf{Z}}\right)$, as the first row in $f_{6}$ because the CSS that is produced by the error is none of the possible error-free CSS.

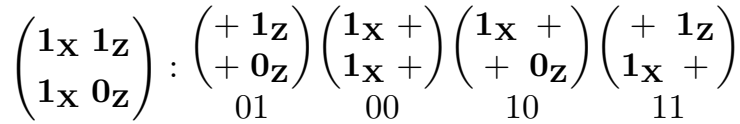

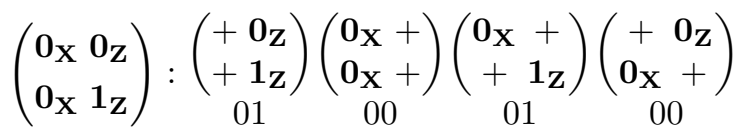

$$
\begin{aligned}
& \text { CSS : } \quad 01010000 \quad 1001 \quad 1100
\end{aligned}
$$

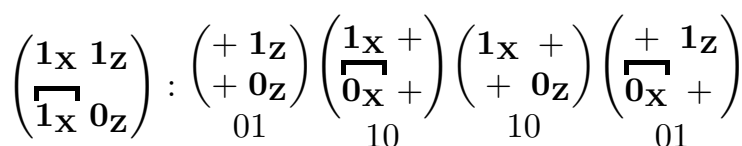

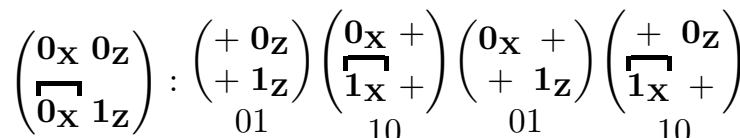

$$
\begin{aligned}
& \text { CSS : } 0101 \quad 1010 \quad 1001 \quad 0110
\end{aligned}
$$

3. The error $\left(\mathbf{0}_{\mathbf{X}}, \mathbf{1}_{\mathbf{Z}}\right)$ is detected with an error-free NO-QP $\left(\mathbf{1}_{\mathbf{X}}, \mathbf{1}_{\mathbf{Z}}\right)$, as the second row in $f_{3}$ because the CSS that is produced by the error is none of the possible error-free CSS.

$$
\begin{aligned}
& \left(\begin{array}{cc}
\mathbf{0}_{\mathbf{X}} & \mathbf{1}_{\mathbf{Z}} \\
\mathbf{1}_{\mathbf{X}} & \mathbf{1}_{\mathbf{Z}}
\end{array}\right): \underset{00}{\left(\begin{array}{c}
+\mathbf{1}_{\mathbf{z}} \\
+\mathbf{1}_{\mathbf{Z}}
\end{array}\right)} \underset{10}{\left(\begin{array}{c}
\mathbf{0}_{\mathbf{X}}+ \\
\mathbf{1}_{\mathbf{X}}+
\end{array}\right)} \underset{01}{\left(\begin{array}{cc}
\mathbf{0}_{\mathbf{X}}+ \\
+ & \mathbf{1}_{\mathbf{Z}}
\end{array}\right)} \underset{11}{\left(\begin{array}{c}
+\mathbf{1}_{\mathbf{Z}} \\
\mathbf{1}_{\mathbf{X}}+
\end{array}\right)} \\
& \left(\begin{array}{cc}
\mathbf{1}_{\mathbf{X}} & \mathbf{0}_{\mathbf{Z}} \\
\mathbf{0}_{\mathbf{X}} & \mathbf{0}_{\mathbf{Z}}
\end{array}\right): \underset{00}{\left(\begin{array}{c}
+\mathbf{0}_{\mathbf{Z}} \\
+\mathbf{0}_{\mathbf{Z}}
\end{array}\right)} \underset{10}{\left(\begin{array}{c}
\mathbf{1}_{\mathbf{X}}+ \\
\mathbf{0}_{\mathbf{X}}+
\end{array}\right)} \underset{10}{\left(\begin{array}{c}
\mathbf{1}_{\mathbf{X}}+ \\
+\mathbf{0}_{\mathbf{Z}}
\end{array}\right)} \underset{00}{\left(\begin{array}{c}
+\mathbf{0}_{\mathbf{Z}} \\
\mathbf{0}_{\mathbf{X}}+
\end{array}\right)} \\
& \text { CSS : } \quad 0000 \quad 1010 \quad 0110 \quad 1100
\end{aligned}
$$

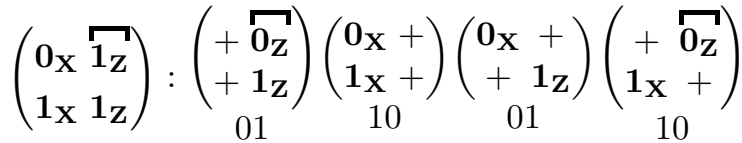

$$
\begin{aligned}
& \left(\begin{array}{cc}
\mathbf{1}_{\mathbf{X}} & \mathbf{0}_{\mathbf{Z}} \\
\mathbf{0}_{\mathbf{X}} & \mathbf{0}_{\mathbf{Z}}
\end{array}\right): \underset{01}{\left(\begin{array}{c}
+\overrightarrow{\mathbf{1}_{\mathbf{Z}}} \\
+\mathbf{0}_{\mathbf{Z}}
\end{array}\right)} \underset{10}{\left(\begin{array}{c}
\mathbf{1}_{\mathbf{X}}+ \\
\mathbf{0}_{\mathbf{x}}+
\end{array}\right)} \underset{10}{\left(\begin{array}{c}
\mathbf{1}_{\mathbf{X}}+ \\
+\mathbf{0}_{\mathbf{z}}
\end{array}\right)} \underset{01}{\left(\begin{array}{c}
+\overparen{\mathbf{1}_{\mathbf{Z}}} \\
\mathbf{0}_{\mathbf{X}}+
\end{array}\right)} \\
& \text { CSS : } \quad 0101 \quad 1010 \quad 0110 \quad 1001
\end{aligned}
$$

4. The error $\left(\mathbf{0}_{\mathbf{X}}, \mathbf{1}_{\mathbf{z}}\right)$ is detected with an error-free NO-QP $\left(\mathbf{1}_{\mathbf{X}}, \mathbf{1}_{\mathbf{z}}\right)$, as the first row in $f_{4}$ because the CSS that is produced by the error is none of the possible error-free CSS. 


$$
\begin{aligned}
& \left(\begin{array}{cc}
\mathbf{1}_{\mathbf{X}} \mathbf{1}_{\mathbf{Z}} \\
\mathbf{0}_{\mathbf{X}} \mathbf{1}_{\mathbf{Z}}
\end{array}\right): \underset{00}{\left(\begin{array}{c}
+\mathbf{1}_{\mathbf{Z}} \\
+\mathbf{1}_{\mathbf{Z}}
\end{array}\right)} \underset{10}{\left(\begin{array}{c}
\mathbf{1}_{\mathbf{X}}+ \\
\mathbf{0}_{\mathbf{X}}+
\end{array}\right)} \underset{11}{\left(\begin{array}{c}
\mathbf{1}_{\mathbf{X}}+ \\
+\mathbf{1}_{\mathbf{Z}}
\end{array}\right)} \underset{01}{\left(\begin{array}{c}
+\mathbf{1}_{\mathbf{z}} \\
\mathbf{0}_{\mathbf{X}}+
\end{array}\right)} \\
& \left(\begin{array}{cc}
\mathbf{0}_{\mathbf{X}} & \mathbf{0}_{\mathbf{Z}} \\
\mathbf{1}_{\mathbf{X}} & \mathbf{0}_{\mathbf{Z}}
\end{array}\right): \underset{00}{\left(\begin{array}{c}
+\mathbf{0}_{\mathbf{Z}} \\
+\mathbf{0}_{\mathbf{Z}}
\end{array}\right)} \underset{0}{\left(\begin{array}{c}
\mathbf{0}_{\mathbf{X}}+ \\
\mathbf{1}_{\mathbf{X}}+
\end{array}\right)} \underset{00}{\left(\begin{array}{c}
\mathbf{0}_{\mathbf{X}}+ \\
+\mathbf{0}_{\mathbf{Z}}
\end{array}\right)} \underset{10}{\left(\begin{array}{c}
+\mathbf{0}_{\mathbf{Z}} \\
\mathbf{1}_{\mathbf{X}}+
\end{array}\right)} \\
& \text { CSS : } \quad 0000 \quad 1010 \quad 1100 \quad 0110
\end{aligned}
$$

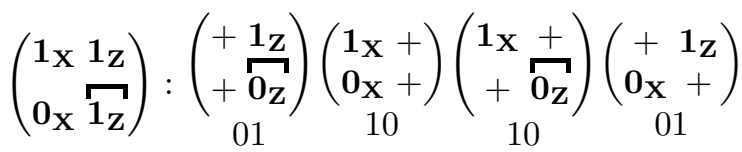

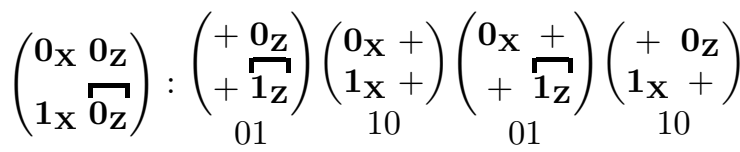

$$
\begin{aligned}
& \text { CSS : } \quad 0101 \quad 1010 \quad 1001 \quad 0110
\end{aligned}
$$

5. The error $\left(\mathfrak{0}_{\mathbf{X}}, \mathbf{1}_{\mathbf{Z}}\right)$ is detected with an error-free NO-QP $\left(\mathbf{0}_{\mathbf{X}}, \mathbf{0}_{\mathbf{Z}}\right)$, as the second row in $f_{9}$ because the CSS that is produced by the error is none of the possible error-free CSS.

$$
\begin{aligned}
& \underset{\left(\begin{array}{ll}
\mathbf{0}_{\mathbf{X}} & \mathbf{1}_{\mathbf{Z}} \\
\mathbf{0}_{\mathbf{X}} & \mathbf{0}_{\mathbf{Z}}
\end{array}\right)}{\left(\begin{array}{c}
+\mathbf{1}_{\mathbf{Z}} \\
+\mathbf{0}_{\mathbf{Z}}
\end{array}\right)} \underset{00}{\left(\begin{array}{c}
\mathbf{0}_{\mathbf{X}}+ \\
\mathbf{0}_{\mathbf{X}}+
\end{array}\right)} \underset{00}{\left(\begin{array}{c}
\mathbf{0}_{\mathbf{X}}+ \\
+\mathbf{0}_{\mathbf{Z}}
\end{array}\right)} \underset{01}{\left(\begin{array}{c}
+\mathbf{1}_{\mathbf{z}} \\
\mathbf{0}_{\mathbf{X}}+
\end{array}\right)}
\end{aligned}
$$

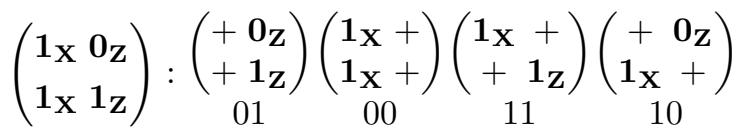

$$
\begin{aligned}
& \begin{array}{lllll}
\text { CSS : } & 0101 & 0000 & 0011 & 0110
\end{array}
\end{aligned}
$$

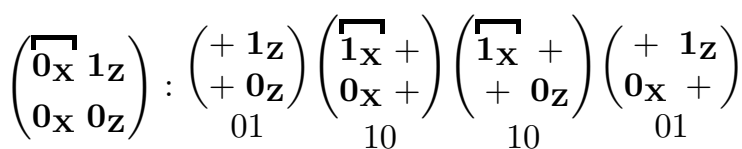

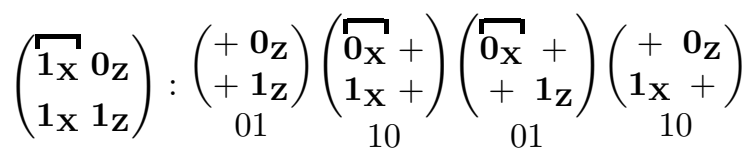

$$
\begin{aligned}
& \text { CSS : } 0101 \quad 1010 \quad 1001 \quad 0110
\end{aligned}
$$

6. The error $\left(\mathbf{1}_{\mathbf{X}}, \mathbf{0}_{\mathbf{Z}}\right)$ is detected with an error-free NO-QP $\left(\mathbf{0}_{\mathbf{X}}, \mathbf{0}_{\mathbf{Z}}\right)$, as the second row in $f_{10}$ because the CSS that is produced by the error is none of the possible error-free CSS. 


$$
\begin{aligned}
& \left(\begin{array}{cc}
\mathbf{1}_{\mathbf{X}} & \mathbf{0}_{\mathbf{Z}} \\
\mathbf{0}_{\mathbf{X}} & \mathbf{0}_{\mathbf{Z}}
\end{array}\right): \underset{00}{\left(\begin{array}{c}
+\mathbf{0}_{\mathbf{Z}} \\
+\mathbf{0}_{\mathbf{Z}}
\end{array}\right)} \underset{0}{\left(\begin{array}{c}
\mathbf{1}_{\mathbf{X}}+ \\
\mathbf{0}_{\mathbf{X}}+
\end{array}\right)} \underset{10}{\left(\begin{array}{c}
\mathbf{1}_{\mathbf{X}}+ \\
+\mathbf{0}_{\mathbf{Z}}
\end{array}\right)} \underset{10}{\left(\begin{array}{c}
+\mathbf{0}_{\mathbf{Z}} \\
\mathbf{0}_{\mathbf{X}}+
\end{array}\right)} \\
& \left(\begin{array}{cc}
\mathbf{0}_{\mathbf{X}} & \mathbf{1}_{\mathbf{Z}} \\
\mathbf{1}_{\mathbf{X}} & \mathbf{1}_{\mathbf{z}}
\end{array}\right): \underset{00}{\left(\begin{array}{c}
+\mathbf{1}_{\mathbf{z}} \\
+\mathbf{1}_{\mathbf{Z}}
\end{array}\right)} \underset{10}{\left(\begin{array}{c}
\mathbf{0}_{\mathbf{X}}+ \\
\mathbf{1}_{\mathbf{X}}+
\end{array}\right)} \underset{01}{\left(\begin{array}{cc}
\mathbf{0}_{\mathbf{X}}+ \\
+ & \mathbf{1}_{\mathbf{Z}}
\end{array}\right)} \underset{11}{\left(\begin{array}{c}
+\mathbf{1}_{\mathbf{z}} \\
\mathbf{1}_{\mathbf{X}}+
\end{array}\right)} \\
& \begin{array}{lllll}
\text { CSS : } & 0000 & 1010 & 1001 & 0011
\end{array}
\end{aligned}
$$

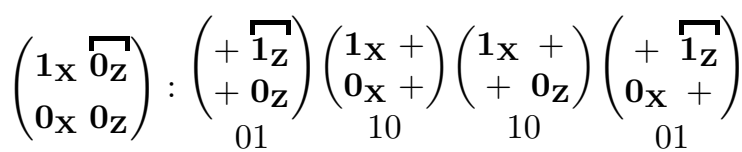

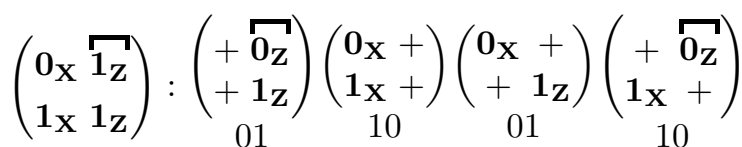

$$
\begin{aligned}
& \text { CSS : } \quad 0101 \quad 1010 \quad 1001 \quad 0110
\end{aligned}
$$

7. The error $\left(\overleftarrow{1}_{\mathbf{X}}, \mathbf{1}_{\mathbf{Z}}\right)$ and also the error $\left(\mathbf{1}_{\mathbf{X}}, \mathfrak{\mathbf { 1 }}_{\mathbf{Z}}\right)$ are detected with an error-free NO-QP $\left(\mathbf{0}_{\mathbf{X}}, \mathbf{0}_{\mathbf{Z}}\right)$, as the first row in $f_{8}$ because the CSS that is produced by the error is none of the possible error-free CSS.

$$
\begin{aligned}
& \left(\begin{array}{cc}
\mathbf{0}_{\mathbf{X}} & \mathbf{0}_{\mathbf{Z}} \\
\mathbf{1}_{\mathbf{X}} & \mathbf{1}_{\mathbf{Z}}
\end{array}\right): \underset{01}{\left(\begin{array}{c}
+\mathbf{0}_{\mathbf{Z}} \\
+\mathbf{1}_{\mathbf{Z}}
\end{array}\right)} \underset{10}{\left(\begin{array}{c}
\mathbf{0}_{\mathbf{X}}+ \\
\mathbf{1}_{\mathbf{X}}+
\end{array}\right)} \underset{01}{\left(\begin{array}{c}
\mathbf{0}_{\mathbf{X}}+ \\
+\mathbf{1}_{\mathbf{Z}}
\end{array}\right)} \underset{10}{\left(\begin{array}{c}
+\mathbf{0}_{\mathbf{Z}} \\
\mathbf{1}_{\mathbf{X}}+
\end{array}\right)} \\
& \left(\begin{array}{cc}
\mathbf{1}_{\mathbf{X}} & \mathbf{1}_{\mathbf{Z}} \\
\mathbf{0}_{\mathbf{X}} & \mathbf{0}_{\mathbf{Z}}
\end{array}\right): \underset{01}{\left(\begin{array}{c}
+\mathbf{1}_{\mathbf{Z}} \\
+\mathbf{0}_{\mathbf{Z}}
\end{array}\right)} \underset{10}{\left(\begin{array}{c}
\mathbf{1}_{\mathbf{X}}+ \\
\mathbf{0}_{\mathbf{X}}+
\end{array}\right)} \underset{10}{\left(\begin{array}{c}
\mathbf{1}_{\mathbf{X}}+ \\
+\mathbf{0}_{\mathbf{Z}}
\end{array}\right)} \underset{01}{\left(\begin{array}{c}
+\mathbf{1}_{\mathbf{Z}} \\
\mathbf{0}_{\mathbf{X}}+
\end{array}\right)} \\
& \begin{array}{lllll}
\text { CSS : } & 0101 & 1010 & 0110 & 1001
\end{array}
\end{aligned}
$$

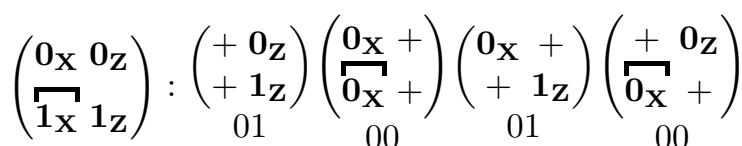

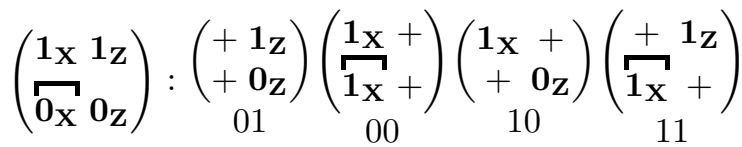

$$
\begin{aligned}
& \text { CSS : } 0101 \quad 0000 \quad 0110 \quad 0011
\end{aligned}
$$

8. The error $\left(\mathfrak{1}_{\mathbf{X}}, \mathbf{1}_{\mathbf{z}}\right)$ and also the error $\left(\mathbf{1}_{\mathbf{X}}, \overparen{\mathbf{1}_{\mathbf{Z}}}\right)$ are detected with an error-free NO-QP $\left(\mathbf{0}_{\mathbf{X}}, \mathbf{0}_{\mathbf{Z}}\right)$, as the second row in $f_{12}$ because the CSS that is produced by the error is none of the possible error-free CSS. 


$$
\begin{aligned}
& \left(\begin{array}{cc}
\mathbf{1}_{\mathbf{X}} \mathbf{1}_{\mathbf{Z}} \\
\mathbf{0}_{\mathbf{X}} & \mathbf{0}_{\mathbf{Z}}
\end{array}\right): \underset{01}{\left(\begin{array}{c}
+\mathbf{1}_{\mathbf{Z}} \\
+\mathbf{0}_{\mathbf{Z}}
\end{array}\right)} \underset{10}{\left(\begin{array}{c}
\mathbf{1}_{\mathbf{X}}+ \\
\mathbf{0}_{\mathbf{X}}+
\end{array}\right)} \underset{10}{\left(\begin{array}{c}
\mathbf{1}_{\mathbf{X}}+ \\
+\mathbf{0}_{\mathbf{Z}}
\end{array}\right)} \underset{01}{\left(\begin{array}{c}
+\mathbf{1}_{\mathbf{Z}} \\
\mathbf{0}_{\mathbf{X}}+
\end{array}\right)} \\
& \left(\begin{array}{cc}
\mathbf{0}_{\mathbf{X}} & \mathbf{0}_{\mathbf{Z}} \\
\mathbf{1}_{\mathbf{X}} & \mathbf{1}_{\mathbf{Z}}
\end{array}\right): \underset{01}{\left(\begin{array}{c}
+\mathbf{0}_{\mathbf{Z}} \\
+\mathbf{1}_{\mathbf{Z}}
\end{array}\right)} \underset{10}{\left(\begin{array}{c}
\mathbf{0}_{\mathbf{X}}+ \\
\mathbf{1}_{\mathbf{X}}+
\end{array}\right)} \underset{01}{\left(\begin{array}{c}
\mathbf{0}_{\mathbf{X}}+ \\
+\mathbf{1}_{\mathbf{Z}}
\end{array}\right)} \underset{10}{\left(\begin{array}{c}
+\mathbf{0}_{\mathbf{Z}} \\
\mathbf{1}_{\mathbf{X}}+
\end{array}\right)} \\
& \begin{array}{lllll}
\text { CSS : } & 0101 & 1010 & 1001 & 0110
\end{array}
\end{aligned}
$$

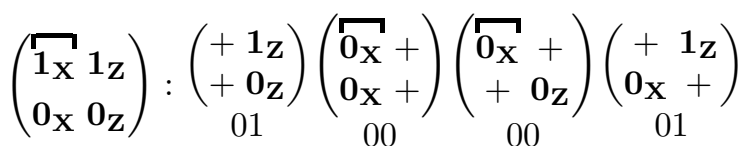

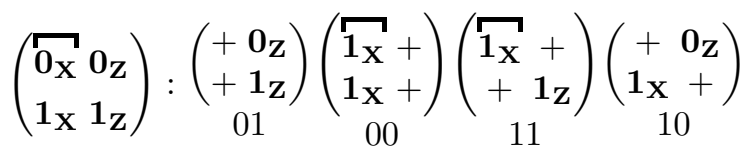

$$
\begin{aligned}
& \text { CSS : } 0101 \quad 0000 \quad 00110110
\end{aligned}
$$

\subsection{Error Correction Pre-processing}

In the error-detection cases exhibited above we have assumed that the non-orthogonal quantum pairs (NO-QP) $\left(\mathbf{0}_{\mathbf{X}}, \mathbf{0}_{\mathbf{Z}}\right)$ and $\left(\mathbf{1}_{\mathbf{X}}, \mathbf{1}_{\mathbf{Z}}\right)$ are error-free, which implies being able to detect any error in these NO-QP. In this section, we explain how to detect such errors using null-frames $f_{7}$ and unitary-frames $f_{11}$. To achieve this we must note that in the absence of error, $f_{7}$ produce CSS that only contain zeros while $f_{11}$ produces 0000 and 1100 under MRT.

Null-frame errors. The error can arrive in several ways and we need a method to detect them, so the following cases can occur:

- Single error:

$$
\left(\begin{array}{ll}
0_{\mathrm{X}} & 0_{\mathrm{Z}} \\
0_{\mathrm{X}} & 0_{\mathrm{z}}
\end{array}\right),\left(\begin{array}{ll}
0_{\mathrm{X}} & 0_{\mathrm{z}} \\
\sigma_{\mathrm{X}} & 0_{\mathrm{z}}
\end{array}\right),\left(\begin{array}{ll}
0_{\mathrm{X}} & 0_{\mathrm{Z}} \\
0_{\mathrm{X}} & 0_{\mathrm{z}}
\end{array}\right),\left(\begin{array}{ll}
0_{\mathrm{X}} & 0_{\mathrm{z}} \\
0_{\mathrm{X}} & 0_{\mathrm{z}}
\end{array}\right)
$$

- Non-orthogonal error, two errors in different basis:

$$
\left(\begin{array}{ccc}
\overrightarrow{0_{\mathrm{X}}} & 0_{\mathrm{Z}} \\
\mathbf{0}_{\mathrm{X}} & \mathbf{0}_{\mathrm{Z}}
\end{array}\right),\left(\begin{array}{cc}
0_{\mathrm{X}} & 0_{\mathrm{Z}} \\
\mathbf{0}_{\mathrm{X}} & 0_{\mathrm{Z}}
\end{array}\right)
$$

- Parallel error, two errors in the same basis:

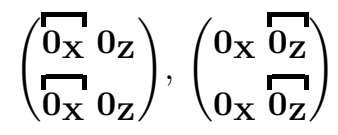

As we will see right away, single and parallel errors will be detected as if they were non-orthogonal error using the algorithm for Detection of Parallel-Pair Errors (DPPE) that we explain next: 
- Alice separates CSSL from null frames into the error-detected-null-frames and the error-freenull-frames just checking that $\mathrm{CSS} \neq 0000$. The last list contain, however, frames with hidden (parallel) errors. For example, consider Alice's null frame $\left(\begin{array}{ll}\mathbf{0}_{\mathbf{X}} & \mathbf{0}_{\mathbf{Z}} \\ \mathbf{0}_{\mathbf{X}} & \mathbf{0}_{\mathbf{Z}}\end{array}\right)$. If Bob's frame contains two errors, say $\left(\begin{array}{l}\mathbf{1}_{\mathbf{X}}+ \\ \mathbf{1}_{\mathbf{X}}+\end{array}\right)$ then the errors kept hidden since $\left(1100, x_{1}, x_{2}\right)$. Alice takes the row-indices $x_{1}$ and $x_{2}$ and she looks for them into the error-detected list (see Table 3).

- Two cases in the error-detected list reveals the errors in $x_{1}$ and $x_{2}$. The frame $\left(\begin{array}{l}\mathbf{1}_{\mathbf{X}}+ \\ \mathbf{0}_{\mathbf{X}}+\end{array}\right)$ with $\left(1010, x_{1}, *\right)$ where the first row is $x_{1}$, reveals an error in the frame but the result it is ambiguous because the frame $\left(\begin{array}{l}\mathbf{0}_{\mathbf{X}}+ \\ \mathbf{1}_{\mathbf{X}}+\end{array}\right)$ also produces CSS=1010. Thus, the result is inconclusive because the CSS does not indicate if the error is in the first or the second row. However, Alice keeps searching into the list of errors and she finds $\left(1100, x_{1}, *\right)$ which comes from the frame $\left(\begin{array}{cc}\mathbf{1}_{\mathbf{X}} & + \\ + & \mathbf{1}_{\mathbf{X}}\end{array}\right)$ where the first row contains $x_{1}$ and the second row also contains an error. Interestingly, this CSS reveals the presence of a non-orthogonal error. Similarly, Alice finds another label that exhibits $x_{2}$ and the parallel error is detected using a non-orthogonal error which applies for single errors too.

The following aspects must be remarked here:

1. Given an error rate in the quantum channel, it is to be expected that about half of the errors will occur in the first quantum state of $\left(\widetilde{\mathbf{0}_{\mathbf{X}}}, \mathbf{0}_{\mathbf{Z}}\right)$ and the other half in the second state $\left(\mathbf{0}_{\mathbf{X}}, \overrightarrow{\mathbf{0}_{\mathbf{Z}}}\right)$. Therefore, the method described to detect single and parallel errors in null-frames is completely feasible.

2. The algorithm detailed above allows finding all the errors in the null frames, but it does not tell us which of the two non-orthogonal states is the error. To find the position of the error, Alice must use an error free NO-QP say $y_{1}=\left(\mathbf{0}_{\mathbf{X}}, \mathbf{0}_{\mathbf{Z}}\right)$ from the list of error-free-null-frames. Then she finds $\left(0101, x_{1}, y_{1}\right)$ which reveals the error is in the first state while $\left(1010, x_{1}, y_{1}\right)$ unveils the error in the second state (see Table 3).

How should it be clear, in all cases, Alice must be able to identify the position of the error to perform reconciliation successfully. Importantly here is that detecting the position of the error also allows Alice to find MR when using this error-row inside a frame. For this purpose and assuming Alice has detected all the errors, consider the following frame cases:

1. First and second rows without errors. Alice applies the usual frame-based sifting algorithm identifying MR in each case.

2. First and second rows with errors. Since error-detection reveals the position of the error, Alice identifies MR straightforward.

3. Error-free (first/second) row and error-detected (second/first) row. In the next lines we discuss this case. 
Suppose Alice has detected all the errors and she must guess MR when she sends $\left(\begin{array}{ll}\mathbf{0}_{\mathbf{X}} & \mathbf{1}_{\mathbf{Z}} \\ \mathbf{0}_{\mathbf{X}} & \mathbf{0}_{\mathbf{Z}}\end{array}\right)$ and Bob gets $\left(\begin{array}{c}+\mathbf{1}_{\mathbf{Z}} \\ \mathbf{1}_{\mathbf{X}}+\end{array}\right)$ thus he returns to Alice CSS=1100. But Alice knows the following facts:

- CSS comes from $f_{9}$.

- The first row is error-free but the first state of the second row is error-detected, that is $\left(\mathbf{0}_{\mathbf{X}}, \mathbf{0}_{\mathbf{Z}}\right)$.

Then Alice tests $f_{9}$ under MRT (see Table 1$)$ given $\left(\mathbf{0}_{\mathbf{X}}, \mathbf{0}_{\mathbf{Z}}\right)$ and $\mathrm{CSS}=1100$, thus she concludes that the unique MR that matches CSS is under $\mathrm{MR}=11$.

Table 3: Analysis of $f_{7}$ frame set. Separate lists of error-detected-null-frames and error-free-null-frames.

The $\mathrm{symbol} *$ represents an arbitrary NO-QP index.

\begin{tabular}{ccc|c|ccc|c}
\multicolumn{9}{c|}{ error-detected } & error-free & comment \\
\hline CSS ! 0 & $i_{1}$ & $i_{2}$ & comment & CSS $=0$ & $i_{1}$ & $i_{2}$ & comm \\
\hline 1010 & $*$ & $*$ & & 0000 & $x_{1}$ & $x_{2}$ & hidden error \\
1100 & $x_{1}$ & $*$ & double-error-detection & 0000 & $y_{1}$ & $y_{2}$ & \\
1100 & $x_{2}$ & $*$ & double-error-detection & 0000 & $*$ & $*$ & \\
1010 & $*$ & $*$ & & 0000 & $*$ & $*$ & \\
1001 & $*$ & $*$ & & 0000 & $*$ & $*$ & \\
0101 & $x_{1}$ & $y_{1}$ & first-state-error & 0000 & $*$ & $*$ & \\
1010 & $x_{2}$ & $y_{1}$ & second-state-error & 0000 & $*$ & $*$ & \\
\hline
\end{tabular}

Unitary-frame errors. Frames $f_{11}$ behave similarly as frames $f_{7}$, so we present the summary results in Table 4 . As far as we go, we are able to detect $\left(\mathbf{0}_{\mathbf{X}}, \mathbf{0}_{\mathbf{Z}}\right),\left(\mathbf{0}_{\mathbf{X}}, \mathbf{0}_{\mathbf{Z}}\right),\left(\mathbf{1}_{\mathbf{X}}, \mathbf{1}_{\mathbf{Z}}\right),\left(\mathbf{1}_{\mathbf{X}}, \mathbf{1}_{\mathbf{Z}}\right)$ errors.

Table 4: Analysis of $f_{11}$ frame set. Separate lists of error-detected-unitary-frames and error-free-unitary-frames. The symbol $*$ represents an arbitrary NO-QP index.

\begin{tabular}{ccc|c|ccc|c}
\multicolumn{9}{c}{ error-detected } & \multicolumn{5}{c}{ error-free } \\
CSS & $i_{1}$ & $i_{2}$ & comment & CSS & $i_{1}$ & $i_{2}$ & comment \\
\hline 1010 & $*$ & $*$ & & 0000 & $x_{1}$ & $x_{2}$ & hidden error \\
0011 & $x_{1}$ & $*$ & double-error-detection & 1100 & $y_{1}$ & $y_{2}$ & \\
0011 & $x_{2}$ & $*$ & double-error-detection & 1100 & $*$ & $*$ & \\
0101 & $*$ & $*$ & & 1100 & $*$ & $*$ & \\
1001 & $*$ & $*$ & & 0000 & $*$ & $*$ & \\
1010 & $x_{1}$ & $y_{1}$ & first-state-error & 0000 & $*$ & $*$ & \\
1001 & $x_{2}$ & $y_{1}$ & second-state-error & 1100 & $*$ & $*$ & \\
\hline
\end{tabular}




\subsection{Reconciliation Algorithm}

To close this section let us summarize the steps of the reconciliation algorithm. Table 5 shows the error detection results using regular frames.

1. Identify $\left(\mathbf{0}_{\mathbf{X}}, \mathbf{0}_{\mathbf{Z}}\right)$ and $\left(\overparen{\mathbf{0}}_{\mathbf{X}}, \mathbf{0}_{\mathbf{Z}}\right),\left(\mathbf{0}_{\mathbf{X}}, \mathfrak{\mathbf { 0 }}_{\mathbf{Z}}\right)$ errors in the set of $f_{7}$. Identify single and parallel errors using DPPE algorithm.

2. Identify $\left(\mathbf{1}_{\mathbf{X}}, \mathbf{1}_{\mathbf{Z}}\right)$ and $\left(\overparen{\mathbf{1}}_{\mathbf{X}}, \mathbf{1}_{\mathbf{Z}}\right),\left(\mathbf{1}_{\mathbf{X}}, \mathbf{1}_{\mathbf{Z}}\right)$ errors in the set of $f_{11}$. Identify single and parallel errors using DPPE algorithm.

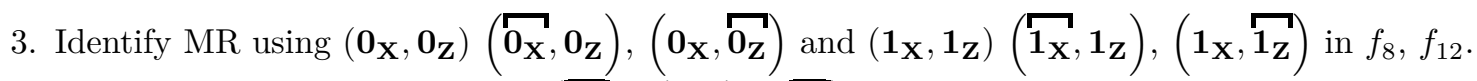

4. Identify $\left(\mathbf{0}_{\mathbf{X}}, \mathbf{1}_{\mathbf{Z}}\right),\left(\mathbf{1}_{\mathbf{X}}, \mathbf{0}_{\mathbf{Z}}\right)$ and $\left(\overparen{\mathbf{0}}_{\mathbf{X}}, \mathbf{1}_{\mathbf{Z}}\right),\left(\mathbf{1}_{\mathbf{X}}, \stackrel{\mathbf{0}_{\mathbf{Z}}}{)}\right)$ errors in $f_{9}, f_{10}, f_{13}, f_{14}$ using $\left(\mathbf{0}_{\mathbf{X}}, \mathbf{0}_{\mathbf{Z}}\right)$, $\left(\overparen{\mathbf{0}}_{\mathbf{X}}, \mathbf{0}_{\mathbf{Z}}\right),\left(\mathbf{0}_{\mathbf{X}}, \overparen{\mathbf{0}}_{\mathbf{Z}}\right)$. Identify MR in $f_{9}, f_{10}, f_{13}, f_{14}$.

5. Identify $\left(\mathbf{0}_{\mathbf{X}}, \mathbf{1}_{\mathbf{Z}}\right),\left(\mathbf{1}_{\mathbf{X}}, \mathbf{0}_{\mathbf{Z}}\right)$ and $\left(\mathbf{0}_{\mathbf{X}}, \boldsymbol{\mathbf { 1 }}_{\mathbf{Z}}\right),\left(\boldsymbol{1}_{\mathbf{X}}, \mathbf{0}_{\mathbf{Z}}\right)$ errors in $f_{2}, f_{6}, f_{3}, f_{4}$ using $\left(\mathbf{1}_{\mathbf{X}}, \mathbf{1}_{\mathbf{Z}}\right)$, $\left(\mathbf{1}_{\mathbf{X}}, \mathbf{1}_{\mathbf{z}}\right),\left(\mathbf{1}_{\mathbf{X}}, \overrightarrow{\mathbf{1}_{\mathbf{Z}}}\right),\left(\mathbf{0}_{\mathbf{X}}, \mathbf{1}_{\mathbf{z}}\right),\left(\mathbf{1}_{\mathbf{X}}, \mathbf{0}_{\mathbf{z}}\right),\left(\overrightarrow{\mathbf{0}_{\mathbf{X}}}, \mathbf{1}_{\mathbf{z}}\right),\left(\mathbf{1}_{\mathbf{X}}, \overrightarrow{\mathbf{0}_{\mathbf{Z}}}\right)$. Identify MR in $f_{2}, f_{6}, f_{3}, f_{4}$.

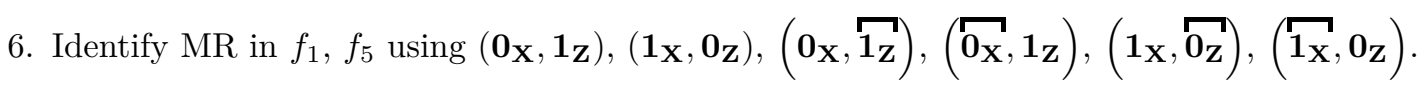

Table 5: Error detection using regular frames.

\begin{tabular}{|c|c|c|c|}
\hline CSS & frame & MR & error detection \\
\hline 1010 & $f_{2}, f_{6}$ & 00 & $\left(\boldsymbol{1}_{\mathbf{x}}, \mathbf{0}_{\mathrm{z}}\right)$ \\
\hline 0110 & $\begin{array}{l}f_{2} \\
f_{6}\end{array}$ & $\begin{array}{l}10 \\
11\end{array}$ & $\left(\overrightarrow{1_{\mathbf{X}}}, 0_{z}\right)$ \\
\hline 1001 & $\begin{array}{l}f_{3} \\
f_{4} \\
\end{array}$ & $\begin{array}{l}11 \\
10 \\
\end{array}$ & $\left(\mathbf{0}_{\mathrm{x}}, \mathbf{\mathbf { 1 } _ { \mathrm { z } }}\right)$ \\
\hline 0101 & $f_{3}, f_{4}$ & 01 & $\left(\mathbf{0}_{\mathbf{x}}, \overline{\mathbf{1}_{\mathrm{z}}}\right)$ \\
\hline $\begin{array}{l}1010 \\
1001\end{array}$ & $f_{9}$ & $\begin{array}{l}00 \\
10\end{array}$ & $\left(\widehat{0_{x}}, 1_{z}\right)$ \\
\hline $\begin{array}{l}1010 \\
1001\end{array}$ & $f_{13}$ & $\begin{array}{l}00 \\
11\end{array}$ & $\left(\overrightarrow{\mathbf{0}_{\mathrm{X}}}, \mathbf{1}_{\mathrm{z}}\right)$ \\
\hline $\begin{array}{l}0101 \\
1001\end{array}$ & $f_{10}$ & $\begin{array}{l}01 \\
10\end{array}$ & $\left(1_{x}, \widetilde{0_{z}}\right)$ \\
\hline $\begin{array}{l}0101 \\
0110\end{array}$ & $f_{14}$ & $\begin{array}{l}01 \\
10\end{array}$ & $\overline{\left(1_{\mathbf{x}}, \overline{0_{z}}\right)}$ \\
\hline
\end{tabular}

As has been demonstrated so far, errors can be detected regardless of the number of errors. Thus, the gain of the secret bits does not depend on the error rate of the quantum channel. 


\section{The Throughput of Frame Reconciliation}

Not all the frames are converted into secret bits. In [23] we derived the throughput as $\frac{1}{4}\left(\frac{1}{2}(1-e)+\frac{1}{6} e\right)$ that reaches a maximum gain of $\frac{1}{8}\left(\begin{array}{l}n \\ 2\end{array}\right)$ when $e=0$. Taking into account Table 2, we arrive to the throughput Equation 1 of frame reconciliation $T$.

$$
\begin{aligned}
T & =\frac{1}{16}\left(4 \cdot \frac{1}{2}+8 \cdot \frac{3}{4}\right)\left(\begin{array}{l}
n \\
2
\end{array}\right) \\
& =\frac{1}{2}\left(\begin{array}{l}
n \\
2
\end{array}\right)=\frac{1}{2} \frac{n(n-1)}{2} \\
& \sim \frac{1}{4} n^{2}
\end{aligned}
$$

Computing the photonic gain of double detection events at Bob's side as $Q_{(+,+)}=\left(1-\mathrm{e}^{-\mu}\right)^{2}$ (neglecting the losses generated by the quantum channel and the losses of the optical detection system), we derived Equation T.1 where $N$ is the total number of quantum pulses sent by Alice to Bob. As a result, the number of secret bits grows doubly quadratically as a function of the number of quantum pulses $N$.

$$
T=\frac{1}{4}\left(1-\mathrm{e}^{-\mu}\right)^{4} N^{4}
$$

One of the biggest challenges posed by the Photon Number Splitting (PNS) attack is that the number of photons per pulse $(\mu)$ should not be increased because an attacker can split the pulse and store a copy of it. However, in frame-based reconciliation, the secret bits do not result only from the quantum pulses that arrive to Bob's detector, but from the double detection events that occur at Bob's station. So the security of our approach does no depend on the photon mean $\mu$ of the quantum pulse neither the channel error-rate $e$.

\section{Immunity to Quantum Attacks}

Produce a double detection event does not depend on the transmittance of the quantum channel but in the quantum probability. This property gives immunity to the quantum key distribution protocol when it relies on double detection event as the vehicle to transmit a secret bit. Let us briefly summarize why the frame-based QKD is immune to the PNS and IR attack (other attack as the basis choice is described in [23]).

On the other hand, reconciliation does not depend on the error rate of the channel, thus Eve cannot hide herself in the noise of the quantum channel.

The Intercept and Resend Attack (IR). Eve must measure each pair of non-orthogonal quantum pulses that cross the quantum channel, then according to the result obtained from her measurement, Eve sends another pair of non-orthogonal quantum pulses to Bob. In addition, Eve must ensure that both states that she forwards to Bob's station are not lost on the quantum channel but assuming she can overcome this difficulty, Eve's final gain is $\frac{1}{4}$. 
Perfect Reconciliation in Quantum Key Distribution with Order-Two Frames

The Photon Number Splitting Attack (PNS). Eve obtains a copy of the quantum states that Bob receives in his optical station and stores them in a quantum memory. However, the probability that Eve gets a double matching detection event is $\frac{1}{2}$. In addition, Eve must measure by choosing between two different measurement bases $(\mathbf{X}$ or $\mathbf{Z})$, so the final gain of the attack is $\frac{1}{4}$.

We must highlight that one the main advantages of the described immunity to PNS and IR quantum attacks, is that the mean photon value $\mu$ in Equation T.1 can be properly increased in the quantum regime, so that longer distance can be achieved in QKD link. Further on, the number of secret bits grows doubly quadratic in the number of quantum pulses.

\section{Conclusions}

We introduced a method to achieve complete reconciliation in Quantum Key Distribution which identifies the transmitted errors in a reverse reconciliation that corrects $100 \%$ of the errors that is invariant with respect to the error rate of the quantum channel.

In the new reconciliation method we compute the Composed Sifting String (CSS) through conjugate frames on Bob's side, which allows Alice to identify and get the position of the errors. By means of the progressive identification of the errors and the specific position of the same within each orthogonal pair, we can obtain almost all MR of the regular frames and from here the secret shared bits.

At least theoretically, the number of secret bits grows doubly quadratically and doubly cubically as a function of the number of quantum pulses sent by Alice because the mean photon value can be properly increased in the quantum regime, so that longer distance can be achieved in QKD link. 


\section{Bibliography}

[1] G. Alagic, J. Alperin-Sheriff, D. Apon, D. Cooper, Q. Dang, J. Kelsey, Y.-K. Liu, C. Miller, D. Moody, R. Peralta, et al., "Status report on the second round of the nist post-quantum cryptography standardization process," US Department of Commerce, NIST, 2020.

[2] A. Wiesmaier, N. Alnahawi, T. Grasmeyer, J. Geißler, A. Zeier, P. Bauspieß, and A. Heinemann, "On pqc migration and crypto-agility," arXiv preprint arXiv:2106.09599, 2021.

[3] L. A. Lizama-Pérez, J. M. López, E. De Carlos-López, and S. E. Venegas-Andraca, "Quantum flows for secret key distribution in the presence of the photon number splitting attack," Entropy, vol. 16, no. 6, pp. 3121-3135, 2014.

[4] L. A. Lizama-Pérez, J. M. López, and E. De Carlos López, "Quantum key distribution in the presence of the intercept-resend with faked states attack," Entropy, vol. 19, no. 1, p. 4, 2016.

[5] M. Mehic, M. Niemiec, H. Siljak, and M. Voznak, "Error reconciliation in quantum key distribution protocols.," 2020.

[6] C. H. Bennett, F. Bessette, G. Brassard, L. Salvail, and J. Smolin, "Experimental quantum cryptography," Journal of cryptology, vol. 5, no. 1, pp. 3-28, 1992.

[7] G. Brassard and L. Salvail, "Secret-key reconciliation by public discussion," in Workshop on the Theory and Application of of Cryptographic Techniques, pp. 410-423, Springer, 1993.

[8] T. B. Pedersen and M. Toyran, "High performance information reconciliation for qkd with cascade," arXiv preprint arXiv:1307.7829, 2013.

[9] W. T. Buttler, S. K. Lamoreaux, J. R. Torgerson, G. Nickel, C. Donahue, and C. G. Peterson, "Fast, efficient error reconciliation for quantum cryptography," Physical Review A, vol. 67, no. 5, p. 052303, 2003.

[10] F. Zhao, M. Fu, F. Wang, Y. Lu, C. Liao, and S. Liu, "Error reconciliation for practical quantum cryptography," Optik, vol. 118, no. 10, pp. 502-506, 2007.

[11] H. Yan, X. Peng, X. Lin, W. Jiang, T. Liu, and H. Guo, "Efficiency of winnow protocol in secret key reconciliation," in 2009 WRI World Congress on Computer Science and Information Engineering, vol. 3, pp. 238-242, IEEE, 2009.

[12] Q. Li, Z. Yang, H. Mao, and X. Wang, "Study on scrambling algorithms of error reconciliation in qkd," in 2018 Eighth International Conference on Instrumentation \& Measurement, Computer, Communication and Control (IMCCC), pp. 1363-1367, IEEE, 2018.

[13] R. Gallager, "Ldpc codes," Information Theory and Reliable Communication, vol. 1, 1963.

[14] C. Elliott, A. Colvin, D. Pearson, O. Pikalo, J. Schlafer, and H. Yeh, "Current status of the darpa quantum network," in Quantum Information and computation III, vol. 5815, pp. 138149, International Society for Optics and Photonics, 2005.

[15] S. Watanabe, R. Matsumoto, and T. Uyematsu, "Tomography increases key rates of quantumkey-distribution protocols," Physical Review A, vol. 78, no. 4, p. 042316, 2008.

[16] A. Mink and A. Nakassis, "Ldpc for qkd reconciliation," arXiv preprint arXiv:1205.4977, 2012.

[17] J. Martinez-Mateo, D. Elkouss, and V. Martin, "Key reconciliation for high performance quantum key distribution," Scientific reports, vol. 3, no. 1, pp. 1-6, 2013.

[18] E. Arikan, "Channel polarization: A method for constructing capacity-achieving codes for symmetric binary-input memoryless channels," IEEE Transactions on information Theory, vol. 55, no. 7, pp. 3051-3073, 2009.

[19] P. Jouguet and S. Kunz-Jacques, "High performance error correction for quantum key distribution using polar codes," arXiv preprint arXiv:1204.5882, 2012. 
[20] A. Nakassis and A. Mink, "Polar codes in a qkd environment," in Quantum Information and Computation XII, vol. 9123, p. 912305, International Society for Optics and Photonics, 2014.

[21] H. Yan, T. Ren, X. Peng, X. Lin, W. Jiang, T. Liu, and H. Guo, "Information reconciliation protocol in quantum key distribution system," in 2008 Fourth International Conference on Natural Computation, vol. 3, pp. 637-641, IEEE, 2008.

[22] L. A. Lizama-Perez and J. M. López, "Quantum key distillation using binary frames," Symmetry, vol. 12, no. 6, p. 1053, 2020.

[23] L. A. Lizama-Pérez, E. H. Samperio, et al., "Beyond the limits of shannon's information in quantum key distribution," Entropy, vol. 23, no. 2, p. 229, 2021. 